\title{
Euler Scheme for One-Dimensional SDEs with Time Dependent Reflecting Barriers
}

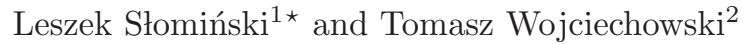 \\ 1 Faculty of Mathematics and Computer Science, Nicolaus Copernicus University, \\ ul. Chopina 12/18, 87-100 Toruń, Poland \\ leszeks@mat . uni . torun.pl \\ 2 Institute of Mathematics and Physics, University of Technology and Agriculture \\ in Bydgoszcz, ul. Al. Prof. S. Kaliskiego 7, 85-796 Bydgoszcz, Poland
}

\begin{abstract}
We give the rate of mean-square convergence for the Euler scheme for one-dimensional stochastic differential equations with time dependent reflecting barriers. Applications to stock prices models with natural boundaries of Bollinger bands type are considered.
\end{abstract}

\section{Introduction}

We consider a market in which fluctuation of stocks prices, and more generally of some economic goods, is given by a stochastic process $S=\left\{S_{t} ; t \in \mathbb{R}^{+}\right\}$ living within the upper- and lower barrier processes $F=\left\{F_{t} ; t \in \mathbb{R}^{+}\right\}$and $G=\left\{G_{t} ; t \in \mathbb{R}^{+}\right\}$, i.e. $G_{t} \leq S_{t} \leq F_{t}, t \in \mathbb{R}^{+}$. Such models appear for instance if some institutions may want to prevent prices from leaving interval $\left[G_{t}, F_{t}\right]$ and prices may have some natural boundaries. Recently, in 8 the simplest case of constant boundaries of the form $[l, d]$ was considered. In this case an option pricing formula was obtained under the assumption that $S$ is a solution of an appropriate stochastic differential equation (SDE). Models of prices fluctuation considered in practice by quantitive analysts are much more general: barriers are stochastic processes depending on the process $S$. Typical examples of such natural boundaries are the so-called Bollinger bands $F, G$ defined by

$$
F_{t}=A_{t}+\alpha\left(\frac{1}{M} \sum_{j=1}^{M}\left(S_{t-\varepsilon j}-A_{t}\right)^{2}\right)^{1 / 2}, \quad G_{t}=A_{t}-\alpha\left(\frac{1}{M} \sum_{j=1}^{M}\left(S_{t-\varepsilon j}-A_{t}\right)^{2}\right)^{1 / 2}
$$

and trading bands (envelopes) defined by

$$
F_{t}=(1+\alpha) A_{t}, \quad G_{t}=(1-\alpha) A_{t},
$$

where $A$ is a moving average process $A_{t}=\frac{1}{M} \sum_{j=1}^{M} S_{t-\varepsilon j}, t \in \mathbb{R}^{+}$, and $\varepsilon, \alpha>0$, $M \in \mathbb{N}$ are some parameters.

In [11] existence and uniqueness of solutions of SDE with time dependent reflecting barriers driven by a general semimartingale is proved. In the present

\footnotetext{
* Research supported by Komitet Badań Naukowych under grant 1 P03A 02226
} 
paper we restrict ourselves to a one-dimensional SDE with reflecting barriers of the form

$$
X_{t}=X_{0}+\int_{0}^{t} \sigma\left(X_{s}\right) d W_{s}+\int_{0}^{t} b\left(X_{s}\right) d s+K_{t}, \quad t \in \mathbb{R}^{+},
$$

where $X_{0} \in \mathbb{R}, W=\left\{W_{t} ; t \in \mathbb{R}^{+}\right\}$is a standard Wiener process, $\sigma, b: \mathbb{R} \rightarrow \mathbb{R}$ are Lipschitz continuous functions and barrier processes $F, G$ are general Lipschitz operators with delayed argument depending possibly on $X$ (for a precise definition see Section 2). Our aim is to define the Euler scheme $\left\{\bar{X}^{n}\right\}$ for the SDE (1) and to give its rate of mean-square convergence.

The main result of the paper says that under mild assumptions on reflecting barrier processes $G\left({ }^{\varepsilon} X\right), F\left({ }^{\varepsilon} X\right)$ with delayed argument for every $q \in \mathbb{R}^{+}$there exists $C>0$ such that

$$
E \sup _{t \leq q}\left|\bar{X}_{t}^{n}-X_{t}\right|^{2} \leq C\left(\frac{\ln n}{n}+E \omega_{1 / n}^{2}\left(G\left({ }^{\varepsilon} X\right), q\right)+E \omega_{1 / n}^{2}\left(F\left({ }^{\varepsilon} X\right), q\right)\right),
$$

where $\omega_{\delta}(x, q)=\sup \left\{\left|x_{t}-x_{s}\right|, 0 \leq s<t \leq q, t-s<\delta\right\}$, for all $\delta>0, q \in \mathbb{R}^{+}$ and $x \in \mathbb{D}\left(\mathbb{R}^{+}, \mathbb{R}\right)\left(\mathbb{D}\left(\mathbb{R}^{+}, \mathbb{R}\right)\right.$ is the space of all mappings $x: \mathbb{R}^{+} \rightarrow \mathbb{R}$ which are right continuous and admit left-hand limits).

From (2) we deduce that in both cases of Bollinger and trading bands for every $q \in \mathbb{R}^{+}, \delta>0$ there exists $C>0$ such that

$$
E \sup _{t \leq q}\left|\bar{X}_{t}^{n}-X_{t}\right|^{2} \leq C\left(\frac{1}{n^{1-\delta}}\right)
$$

Moreover, in both cases,

$$
E \sup _{t \leq q}\left|\bar{X}_{t}^{n}-X_{t}\right|^{2} \leq C\left(\frac{\ln n}{n}\right)
$$

if $\sigma, b$ are bounded.

Note that if $G=-\infty, F=+\infty$ then $\left\{\bar{X}^{n}\right\}$ is the classical Euler scheme introduced in [6]. In the case $G=0, F=+\infty$ and $G=l, F=d$ the rate of mean-square convergence was examined earlier by many authors (see, e.g., [2,4] $5,7.910]$ ).

In the paper no attemps has been made to obtain option pricing formulas for markets with dynamics of prices given by (11). This question deserves an independent study.

\section{SDEs with Time Dependent Reflecting Barriers}

We begin with a definition of the Skorokhod problem with time dependent reflecting barriers.

Definition 1. Let $y, f, g \in \mathbb{D}\left(\mathbb{R}^{+}, \mathbb{R}\right)$ with $g \leq f$ and $g_{0} \leq y_{0} \leq f_{0}$. We say that a pair $(x, k) \in \mathbb{D}\left(\mathbb{R}^{+}, \mathbb{R}^{2}\right)$ is a solution of the Skorokhod problem associated with $y$ and barriers $f, g$ (and write $(x, k)=S P(y, f, g))$ if 
(i) $x_{t}=y_{t}+k_{t}, t \in \mathbb{R}^{+}$,

(ii) $g_{t} \leq x_{t} \leq f_{t}, t \in \mathbb{R}^{+}$.

(iii) $k_{t}=k_{t}^{(-)}-k_{t}^{(+)}, t \in \mathbb{R}^{+}$, where $k^{(-)}, k^{(+)}$are nondecreasing, right continuous functions with $k_{0}=k_{0}^{(-)}=k_{0}^{(+)}=0$ such that $k^{(-)}$increases only on $\left\{t ; x_{t}=g_{t}\right\}$ and $k^{(+)}$increases only on $\left\{t ; x_{t}=f_{t}\right\}$.

Theorem 1. ([11] ) Assume that $f, g \in \mathbb{D}\left(\mathbb{R}^{+}, \mathbb{R}\right)$ satisfy the condition

$$
\inf _{t \leq q}\left(f_{t}-g_{t}\right)>0, \quad q \in \mathbb{R}^{+} .
$$

Then for every $y \in \mathbb{D}\left(\mathbb{R}^{+}, \mathbb{R}\right)$ with $g_{0} \leq y_{0} \leq f_{0}$, there exists a unique solution $(x, k)$ of the Skorokhod problem associated with $y$ and barriers $f, g$.

Note that in the case of continuous function $y$ similar definitions of the Skorokhod problem were earlier given in [3] and [1]. These papers contain also results on existence and uniqueness of solutions of the Skorokhod problem in the case of continuous $y$ and continuous barriers $f, g$ (see e.g. [3. Lemma 4.1]).

The following theorem, where Lipschitz continuity of solutions of the Skorokhod problem is stated will prove to be very useful in Section 3.

Theorem 2. ([11]) Assume that $y^{i}, f^{i}, g^{i} \in \mathbb{D}\left(\mathbb{R}^{+}, \mathbb{R}\right), g_{0}^{i} \leq y_{0}^{i} \leq f_{0}^{i}$ and

$$
\inf _{t \leq q}\left(f_{t}^{i}-g_{t}^{i}\right)>0, \quad q \in \mathbb{R}^{+}
$$

for $i=1,2$. Let $\left(x^{i}, k^{i}\right)=S P\left(y^{i}, f^{i}, g^{i}\right), i=1,2$. Then for every $q \in \mathbb{R}^{+}$

$$
\sup _{t \leq q}\left|x_{t}^{1}-x_{t}^{2}\right| \leq 3 \sup _{t \leq q}\left|y_{t}^{1}-y_{t}^{2}\right|+\sup _{t \leq q}\left|f_{t}^{1}-f_{t}^{2}\right|+\sup _{t \leq q}\left|g_{t}^{1}-g_{t}^{2}\right|
$$

and

$$
\sup _{t \leq q}\left|k_{t}^{1}-k_{t}^{2}\right| \leq 2 \sup _{t \leq q}\left|y_{t}^{1}-y_{t}^{2}\right|+\sup _{t \leq q}\left|f_{t}^{1}-f_{t}^{2}\right|+\sup _{t \leq q}\left|g_{t}^{1}-g_{t}^{2}\right|
$$

Let $\left(\Omega, \mathcal{F},\left(\mathcal{F}_{t}\right), P\right)$ be a filtered probability space.

Definition 2. Let $\mathcal{D}$ denote the space of all $\left(\mathcal{F}_{t}\right)$ adapted processes with trajectories in $\mathbb{D}\left(\mathbb{R}^{+}, \mathbb{R}\right)$. We say that an operator $H: \mathcal{D} \rightarrow \mathcal{D}$ is Lipschitz if

(i) $H(X) \in \mathcal{D}$ for any $X \in \mathcal{D}$,

(ii) for any $X, Y \in \mathcal{D}$ and any stopping time $\tau$,

$X^{\tau-}=Y^{\tau-}$ implies $H(X)^{\tau-}=H(Y)^{\tau-}$,

(iii) there exists $L>0$ such that for any $X, Y \in \mathcal{D}$

$$
\left|H(X)_{t}-H(Y)_{t}\right| \leq L \sup _{s \leq t}\left|X_{t}-Y_{t}\right|, \quad t \in \mathbb{R}^{+} .
$$


Given $X \in \mathcal{D}$ and $\varepsilon>0$ set ${ }^{\varepsilon} X=\left\{X_{t-\varepsilon} ; t \in \mathbb{R}^{+}\right\}$(with the convention that $X_{t}=X_{0}$ for $t \in[-\varepsilon, 0)$ ). In what follows barriers of the form $F\left({ }^{\varepsilon} X\right), G\left({ }^{\varepsilon} X\right)$, where $F, G$ are Lipschitz operators we will call barrier operators with delayed arguments.

Fix $\varepsilon>0$. Let $W$ be an $\left(\mathcal{F}_{t}\right)$ adapted Wiener process and let $F, G$ be two Lipschitz operators such that for any $X \in \mathcal{D}$

$$
\inf _{t \leq q}\left(F\left({ }^{\varepsilon} X\right)_{t}-G\left({ }^{\varepsilon} X\right)_{t}\right)>0, \quad q \in \mathbb{R}^{+} .
$$

We will say that a pair $(X, K)$ of $\left(\mathcal{F}_{t}\right)$ adapted processes is a strong solution of the SDE (1) with barrier operators $F\left({ }^{\varepsilon} X\right), G\left({ }^{\varepsilon} X\right)$ with delayed argument if $(X, K)=S P\left(Y, F\left({ }^{\varepsilon} X\right), G\left({ }^{\varepsilon} X\right)\right)$, where

$$
Y_{t}=X_{0}+\int_{0}^{t} \sigma\left(X_{s}\right) d W_{s}+\int_{0}^{t} b\left(X_{s}\right) d s, \quad t \in \mathbb{R}^{+} .
$$

Theorem 3. (11]) Let $\varepsilon>0$. Assume that $\sigma, b$ are Lipschitz continuous functions and F, $G$ are Lipschitz operators satisfying (3) with $G\left(X_{0}\right)_{0} \leq X_{0} \leq$ $F\left(X_{0}\right)_{0}$. Then there exists a unique strong solution $(X, K)$ of the SDE (1).

Theorem 4. Let $\varepsilon>0$ and let $\sigma, b, F, G, X_{0}$ satisfy the assumptions of Theorem 3. If

$$
E \sup _{t \leq q}\left(\left|F\left(X_{0}\right)_{t}\right|+\left|G\left(X_{0}\right)_{t}\right|\right)^{2 p}<+\infty, \quad q \in \mathbb{R}^{+}, p \in \mathbb{N}
$$

then

(i) $E \sup _{t \leq q}\left|X_{t}-X_{0}\right|^{2 p}<+\infty, p \in \mathbb{N}, q \in \mathbb{R}^{+}$,

(ii) if moreover, $F\left({ }^{\varepsilon} X\right)_{s}=F\left({ }^{\varepsilon} X^{s}\right)_{t}$ and $G\left({ }^{\varepsilon} X\right)_{s}=G\left({ }^{\varepsilon} X^{s}\right)_{t}$ for any $0 \leq s \leq t$, then for every $p \in \mathbb{N}, q \in \mathbb{R}^{+}$there exists $C>0$ such that

$$
E\left|X_{t}-X_{s}\right|^{2 p} \leq C(t-s)^{p}
$$

for $s \leq t \leq q$

Corollary 1. Under assumptions of Theorems 3 and 4 for any $q \in \mathbb{R}^{+}, \delta>0$ there exists $C>0$ such that

$$
E\left(\omega_{1 / n}\left(F\left({ }^{\varepsilon} X\right), q\right)\right)^{2}+E\left(\omega_{1 / n}\left(G\left({ }^{\varepsilon} X\right), q\right)\right)^{2} \leq C\left(\frac{1}{n^{1-\delta}}\right) .
$$

If moreover, $\sigma, b$ are bounded then

$$
E\left(\omega_{1 / n}\left(F\left({ }^{\varepsilon} X\right), q\right)\right)^{2}+E\left(\omega_{1 / n}\left(G\left({ }^{\varepsilon} X\right), q\right)\right)^{2} \leq C\left(\frac{\ln n}{n}\right) .
$$




\section{Euler Scheme for SDEs with Time Dependent Reflecting Barrier Operators}

Let $\left(\mathcal{F}_{t}^{n}\right)$ denote the discretization of $\left(\mathcal{F}_{t}\right)$, i.e. $\mathcal{F}_{t}^{n}=\mathcal{F}_{k / n}$ for $t \in[k / n,(k+1) / n)$ and let $F^{n}, G^{n}$ denote the discretizations of operators $F, G$, i.e. any process $X$, $F^{n}(X)_{t}=F(X)_{k / n}, G^{n}(X)_{t}=G(X)_{k / n}$ for any $t \in[k / n,(k+1) / n)$.

The Euler scheme for the SDE (11) is given by the following recurrent formula

$$
\left\{\begin{aligned}
\bar{X}_{0}^{n}= & X_{0}, \\
\bar{X}_{(k+1) / n}^{n}= & \max \left[\operatorname { m i n } \left(\bar{X}_{k / n}^{n}+\sigma\left(\bar{X}_{k / n}^{n}\right)\left(W_{(k+1) / n}-W_{k / n}\right)\right.\right. \\
& \left.\left.+b\left(\bar{X}_{k / n}^{n}\right) \frac{1}{n}, F\left({ }^{\varepsilon} \bar{X}^{n, k / n}\right)_{(k+1) / n}\right), G\left({ }^{\varepsilon} \bar{X}^{n, k / n}\right)_{(k+1) / n}\right], \\
\bar{X}_{t}^{n} \quad & \bar{X}_{k / n}^{n}, \quad t \in[k / n,(k+1) / n),
\end{aligned}\right.
$$

where ${ }^{\varepsilon} \bar{X}_{t}^{n, k / n}={ }^{\varepsilon} \bar{X}_{t}^{n}$ if $t \leq k / n$ and ${ }^{\varepsilon} \bar{X}_{t}^{n, k / n}={ }^{\varepsilon} \bar{X}_{k / n}^{n}$ if $t>k / n$. Set

$$
\bar{Y}_{t}^{n}=X_{0}+\int_{0}^{t} \sigma\left(\bar{X}_{s-}^{n}\right) d W_{s}^{n}+\int_{0}^{t} b\left(\bar{X}_{s-}^{n}\right) d \rho_{s}^{n}, \quad t \in \mathbb{R}^{+},
$$

where $\rho_{t}^{n}=k / n$ for $t \in[k / n,(k+1) / n)$ and $W^{n}$ is a disretization of Wiener process $W$, that is $W_{t}^{n}=W_{\rho_{t}^{n}}, t \in \mathbb{R}^{+}$. Note that $\left(\bar{X}^{n}, \bar{K}^{n}=\bar{X}^{n}-\bar{Y}^{n}\right)$ is a pair of $\left(\mathcal{F}_{t}^{n}\right)$ adapted processes such that $\left(\bar{X}^{n}, \bar{K}^{n}\right)=S P\left(\bar{Y}^{n}, F^{n}\left({ }^{\varepsilon} \bar{X}^{n}\right), G^{n}\left({ }^{\varepsilon} \bar{X}^{n}\right)\right)$.

Theorem 5. Assume that $\sigma, b$ are Lipschitz continuous functions and $F, G$ are Lipschitz operators satysfying (3) such that $G\left(X_{0}\right)_{0} \leq X_{0} \leq F\left(X_{0}\right)_{0}$

$$
E \sup _{t \leq q}\left(\left|F\left(X_{0}\right)_{t}\right|+\left|G\left(X_{0}\right)_{t}\right|\right)^{2+\delta}<+\infty, \quad q \in \mathbb{R}^{+},
$$

for some $\delta>0$. If $(X, K)$ is a solution of the $S D E$ (11) then for every $q \in \mathbb{R}^{+}$ there exists $C>0$ such that (2) holds true.

Proof. We begin by proving that

$$
\sup _{n} E \sup _{t \leq q}\left|\bar{X}_{t}^{n}-X_{0}\right|^{2+\delta}<\infty
$$

Without loss of generality we may and will assume that

$$
\sup _{n} E \sup _{t \leq q-\varepsilon}\left|\bar{X}_{t}^{n}-X_{0}\right|^{2+\delta}<\infty .
$$

Since $\left(X_{0}, 0\right)=S P\left(X_{0}, F^{n}\left(X_{0}\right)_{0}, G^{n}\left(X_{0}\right)_{0}\right)$, it follows from Theorem 2 that

$$
\begin{aligned}
\sup _{t \leq q}\left|\bar{X}_{t}^{n}-X_{0}\right| \leq & 3 \sup _{t \leq q}\left|\int_{0}^{t} \sigma\left(\bar{X}_{s-}^{n}\right) d W_{s}^{n}+\int_{0}^{t} b\left(\bar{X}_{s-}^{n}\right) d \rho_{s}^{n}\right| \\
& +\sup _{t \leq q}\left(\left|F^{n}\left({ }^{\varepsilon} \bar{X}^{n}\right)_{t}-F^{n}\left(X_{0}\right)_{t}\right|+\left|G^{n}\left({ }^{\varepsilon} \bar{X}^{n}\right)_{t}-G^{n}\left(X_{0}\right)_{t}\right|\right)+H_{q},
\end{aligned}
$$

where $H_{q}=\sup _{t \leq q}\left(\left|G^{n}\left(X_{0}\right)_{t}-G^{n}\left(X_{0}\right)_{0}\right|+\left|F^{n}\left(X_{0}\right)_{t}-F^{n}\left(X_{0}\right)_{0}\right|\right)$. Clearly 


$$
\begin{aligned}
E \sup _{t \leq q}\left(\left|F^{n}\left({ }^{\varepsilon} \bar{X}^{n}\right)_{t}-F^{n}\left(X_{0}\right)_{t}\right|+\right. & \left.\left|G^{n}\left({ }^{\varepsilon} \bar{X}^{n}\right)_{t}-G^{n}\left(X_{0}\right)_{t}\right|\right)^{2+\delta} \\
& \leq C E \sup _{t \leq q-\varepsilon}\left|\bar{X}_{t}^{n}-X_{t}\right|^{2+\delta}<+\infty,
\end{aligned}
$$

and $E H_{q}^{2+\delta}<\infty$. Therefore, by the Burkholder-Davis-Gundy and Hölder inequalities we have

$$
E \sup _{t \leq q}\left|\bar{X}_{t}^{n}-X_{0}\right|^{2+\delta} \leq C\left\{\int_{0}^{q} E \sup _{u \leq s}\left|\bar{X}_{u-}^{n}-X_{0}\right|^{2+\delta} d s+1\right\}
$$

and hence (7). Since $\sigma, b$ are Lipschitz continuous (7) yields

$$
\sup _{n} E \sup _{t \leq q}\left|\sigma\left(\bar{X}^{n}\right)_{t}\right|^{2+\delta}<\infty, \quad \sup _{n} E \sup _{t \leq T}\left|b\left(\bar{X}^{n}\right)_{t}\right|^{2+\delta}<\infty .
$$

Set

$$
\widehat{Y}_{t}^{n}=X_{0}+\int_{0}^{t} \sigma\left(\bar{X}_{s-}^{n}\right) d W_{s}+\int_{0}^{t} b\left(\bar{X}_{s-}^{n}\right) d s, \quad t \in \mathbb{R}^{+},
$$

and $\left(\hat{X}^{n}, \hat{K}^{n}\right)=S P\left(\hat{Y}^{n}, F^{n}\left({ }^{\varepsilon} \bar{X}^{n}\right), G^{n}\left({ }^{\varepsilon} \bar{X}^{n}\right)\right)$. Then

$$
\hat{Y}_{t}^{n}-\bar{Y}_{t}^{n}=\sigma\left(\bar{X}_{k / n}^{n}\right)\left(W_{t}-W_{k / n}\right)+b\left(\bar{X}_{k / n}^{n}\right)(t-k / n)
$$

for $t \in[k / n,(k+1) / n)$. Therefore, by Theorem 2, (8), (9) and [9, Lemma A4]

$$
\begin{aligned}
& E \sup _{t \leq q}\left|\bar{X}_{t}^{n}-\hat{X}_{t}^{n}\right|^{2} \leq 9 E \sup _{t \leq q}\left|\bar{Y}_{t}^{n}-\hat{Y}_{t}^{n}\right|^{2} \\
& \leq 9\left\{\left(E \sup _{t \leq q}\left|\sigma\left(\bar{X}_{t-}^{n}\right)\right|^{2+\delta}\right)^{2 /(2+\delta)}\left(E\left(\omega_{1 / n}(W, q)\right)^{(2 \delta+4) / \delta}\right)^{\delta /(\delta+2)}\right. \\
& \left.\quad+\left(\frac{1}{n}\right)^{2} E \sup _{t \leq q}\left|b\left(\bar{X}_{t-}^{n}\right)\right|^{2}\right\} \\
& \leq C\left(\frac{\ln n}{n}+\left(\frac{1}{n}\right)^{2}\right) \leq C\left(\frac{\ln n}{n}\right) .
\end{aligned}
$$

Clearly

$$
\begin{aligned}
E \sup _{t \leq q}\left|\hat{X}_{t}^{n}-X_{t}\right|^{2} \leq C\left\{E \sup _{t \leq q}\left|\hat{Y}_{t}^{n}-Y_{t}\right|^{2}\right. \\
\quad+E\left(\sup _{t \leq q}\left|F^{n}\left({ }^{\varepsilon} \bar{X}^{n}\right)_{t}-F^{n}\left({ }^{\varepsilon} X\right)_{t}\right|+\left|G^{n}\left({ }^{\varepsilon} \bar{X}^{n}\right)_{t}-G^{n}\left({ }^{\varepsilon} X\right)_{t}\right|\right)^{2} \\
\left.\quad+E\left(\sup _{t \leq q}\left|F^{n}\left({ }^{\varepsilon} X\right)_{t}-F\left({ }^{\varepsilon} X\right)_{t}\right|+\left|G^{n}\left({ }^{\varepsilon} X\right)_{t}-G\left({ }^{\varepsilon} X\right)_{t}\right|\right)^{2}\right\} \\
\leq C\left\{\int_{0}^{q} E \sup _{u \leq s}\left|\bar{X}_{u}^{n}-X_{u}\right|^{2} d s+E \sup _{t \leq q-\varepsilon}\left|\bar{X}_{t}^{n}-X_{t}\right|^{2}+\delta_{n}\right\}
\end{aligned}
$$

where $\delta_{n}=E \omega_{1 / n}^{2}\left(F\left({ }^{\varepsilon} X\right), q\right)+E \omega_{1 / n}^{2}\left(G\left({ }^{\varepsilon} X\right), q\right)$. Since without loss of generality we may and will assume

$$
E \sup _{t \leq q-\varepsilon}\left|\bar{X}_{t}^{n}-X_{t}\right|^{2} \leq C\left(\frac{\ln n}{n}+E \omega_{1 / n}^{2}\left(G\left({ }^{\varepsilon} X\right), q-\varepsilon\right)+E \omega_{1 / n}^{2}\left(F\left({ }^{\varepsilon} X\right), q-\varepsilon\right)\right),
$$

from (10) and (11) we obtain 


$$
\begin{aligned}
E \sup _{t \leq q}\left|\bar{X}_{t}^{n}-X_{t}\right|^{2} & \leq 2 E \sup _{t \leq q}\left|\bar{X}_{t}^{n}-\hat{X}_{t}^{n}\right|^{2}+2 E \sup _{t \leq q}\left|\hat{X}_{t}^{n}-X_{t}\right|^{2} \\
& \leq C\left\{\frac{\ln n}{n}+\int_{0}^{t} E \sup _{u \leq s}\left|\bar{X}_{u}^{n}-X_{u}\right|^{2} d u+\delta_{n}\right\} .
\end{aligned}
$$

To complete the proof it suffices now to use Gronwall's lemma.

Corollary 2. Assume that $\sigma, b$ are Lipschitz continuous functions and $F, G$ are $\left(\mathcal{F}_{t}\right)$ adapted processes such that $G_{0} \leq X_{0} \leq F_{0}$, inf $f_{t \leq q}\left(F_{t}-G_{t}\right)>0, q \in \mathbb{R}^{+}$ and

$$
E \sup _{t \leq q}\left(\left|F_{t}\right|+\left|G_{t}\right|\right)^{2+\delta}<+\infty, \quad q \in \mathbb{R}^{+},
$$

for some $\delta>0$. If $(X, K)$ is a solution of the $S D E$ (1) then for every $q \in \mathbb{R}^{+}$ there exists $C>0$ such that

$$
E \sup _{t \leq q}\left|\bar{X}_{t}^{n}-X_{t}\right|^{2} \leq C\left(\frac{\ln n}{n}+E \omega_{1 / n}^{2}(G, q)+E \omega_{1 / n}^{2}(F, q)\right),
$$

Corollary 3. If $\sigma, b$ are Lipschitz continuous and $F, G$ are Bollinger or trading bands then for every $q \in \mathbb{R}^{+}, \delta>0$ there exists $C>0$ such that

$$
E \sup _{t \leq q}\left|\bar{X}_{t}^{n}-X_{t}\right|^{2} \leq C\left(\frac{1}{n^{1-\delta}}\right) .
$$

Proof. Due to Theorem 5 and (5) it is sufficient to prove that the respective barrier operators $F, G$ are Lipschitz.

First we will consider the case of Bolinger bands. We restrict our attention to the operator $F$. Observe that it has the following form:

$$
F(X)_{t}=B(X)_{t}+\alpha\left(\frac{1}{M} \sum_{j=0}^{M-1}\left(X_{t-\varepsilon j}-B(X)_{t}\right)^{2}\right)^{1 / 2}
$$

where $B(X)_{t}=\frac{1}{M} \sum_{j=0}^{M-1} X_{t-\varepsilon j}, t \in \mathbb{R}^{+}, X \in \mathcal{D}$. From the above formula it follows immediately that $F$ posseses the properties (i) and (ii) of Definition 2 Moreover, for any $X, Y \in \mathcal{D}$ and $t \leq q \in \mathbb{R}^{+}$,

$$
\begin{aligned}
\mid F(X)_{t}- & F(Y)_{t}|\leq| B(X)_{t}-B(Y)_{t} \mid \\
& +\alpha\left|\left(\frac{1}{M} \sum_{j=0}^{M-1}\left(X_{t-\varepsilon j}-B(X)_{t}\right)^{2}\right)^{1 / 2}-\left(\frac{1}{M} \sum_{j=0}^{M-1}\left(Y_{t-\varepsilon j}-B(Y)_{t}\right)^{2}\right)^{1 / 2}\right| \\
\leq & \sup _{t \leq q}\left|X_{t}-Y_{t}\right|+\alpha\left(\frac{1}{M} \sum_{j=0}^{M-1}\left(X_{t-\varepsilon j}-Y_{t-\varepsilon j}+B(X)_{t}-B(Y)_{t}\right)^{2}\right)^{1 / 2} \\
\leq & \sup _{t \leq q}\left|X_{t}-Y_{t}\right|+\alpha \max _{0 \leq j \leq M-1}\left|X_{t-\varepsilon j}-Y_{t-\varepsilon j}+B(X)_{t}-B(Y)_{t}\right| \\
\leq & (1+2 \alpha) \sup _{t \leq q}\left|X_{t}-Y_{t}\right|
\end{aligned}
$$

which shows that $F$ is Lipschitz. 
In the case of trading bands $F$ has the form:

$$
F(X)_{t}=(1+\alpha) B(X)_{t}, \quad t \in \mathbb{R}^{+} .
$$

Hence $F$ posseses properties (i) and (ii) of Definition 2 and for $X, Y \in \mathcal{D}, q \in \mathbb{R}^{+}$ we have

$$
\sup _{t \leq q}\left|F(X)_{t}-F(Y)_{t}\right| \leq(1+\alpha) \sup _{t \leq q}\left|X_{t}-Y_{t}\right|
$$

so $F$ is Lipschitz.

Corollary 4. If $\sigma, b$ are Lipschitz continuous and bounded functions and $F, G$ are Bollinger or trading bands then for every $q \in \mathbb{R}^{+}$there exists $C>0$ such that

$$
E \sup _{t \leq q}\left|\bar{X}_{t}^{n}-X_{t}\right|^{2} \leq C\left(\frac{\ln n}{n}\right) .
$$

Proof. It follows from ([6), Theorem 5 and the fact that in both cases $F, G$ are Lipschitz.

\section{References}

1. K. Burdzy, E. Toby, A Skorokhod-type lemma and a decomposition of reflected Brownian motion, Ann. Probab., 23 (1995), 584-604.

2. R.J. Chitashvili, N.L. Lazrieva, Strong solutions of stochastic differential equations with boundary conditions, Stochastics,5, (1981), 225-309.

3. M. Nagasawa, T. Domenig, Diffusion processes on an open time interval and their time reversal, Itô's stochastic calculus and probability theory, 261-280, Springer, Tokio 1996.

4. G.N. Kinkladze, Thesis, Tbilissi, (1983).

5. D. Lépingle, Euler scheme for reflected stochastic differential equations, Mathematics and Computers in Simulations, 38 (1995), 119-126.

6. G. Maruyama, Continuous Markov processes and stochastic equations, Rend. Circ. Mat. Palermo, 4, (1955), 48-90.

7. R. Pettersson, Approximations for stochastic differential equations with reflecting convex boundaries, Stochastic Process. Appl. 59, (1995), 295-308.

8. S. Rady, Option pricing in the presence of natural boundaries and quadratic diffusion term, Finance and Stochastics, 1 (1997), 331-344.

9. L. Słomiński, Euler's approximations of solutions of SDEs with reflecting boundary, Stochastic Process. Appl., 94, (2001), 317-337.

10. L. Słomiński, On approximation of solutions of multidimensional SDEs with reflecting boundary conditions, Stochastic Process. Appl., 50, (1994), 197-219.

11. L. Słomiński, T. Wojciechowski, One-dimensional stochastic differential equations with time dependent reflecting barriers, submitted (2004). 